\title{
Correlation analysis between the LDL-C in serum and the onset of transient ischemic attack caused by CSVD
}

\author{
YAQI CHEN, MEI HU and HONGYING GONG \\ Department of Neurology, Xiangyang Central Hospital, The Affiliated Hospital of Hubei University \\ of Arts and Science, Xiangyang, Hubei 441021, P.R. China \\ Received December 20, 2016; Accepted May 11, 2017
}

DOI: $10.3892 / \mathrm{etm} .2017 .4583$

\begin{abstract}
The aim of this study was to investigate the correlation between the low-density lipoprotein cholesterol (LDL-C) in serum and the onset of transient ischemic attack caused by cerebral small vascular disease (CSVD). Between September 2012 and September 2015, 249 patients who were diagnosed as CSVD were randomly enrolled in this study. According to MRI results, patients were divided into the patient and control groups. In the patient group, the patients were further subdivided into the white matter lesion (WML) group $(\mathrm{n}=86)$ and lacunar infarction (LI) group $(\mathrm{n}=53)$. Head MRI and/or CT were conducted on all the patients. This included T1 and $\mathrm{T} 2$ phases, diffusion weighted imaging (DWI) and fluid attenuated inversion recovery (FLAIR). Additionally, mini-mental status examination (MMSE) test and Montreal cognitive assessment (MoCA) test were carried out on all the patients. As a result, the age, total cholesterol (TC) level and low-density lipoprotein (LDL) levels in the patient group were higher than those in the control group $(\mathrm{p}<0.05)$. The MMSE and MoCA scores in the patient group were significantly lower than those in the control group $(\mathrm{p}<0.05)$. With all the risk factors being set as independent variables and small vessel disease (SVD) as the dependent variable, we performed the logistic regression analysis and correlation analysis for paired data, and found that the increase in LDL was correlated to the onset of SVD, OR=1,321. After adjustment of other risk factors, we enrolled the level of triglyceride (TG) into the multivariable analysis and obtained a statistically significant difference $(\mathrm{p}<0.05)$. In conclusion, $\mathrm{LDL}$ is a major risk factor affecting the onset of transient ischemic attack (TIA) induced by CSVD. Patients with hyperlipidemia should receive head MRI or CT examination to eliminate the probability of the
\end{abstract}

Correspondence to: Dr Hongying Gong, Department of Neurology, Xiangyang Central Hospital, The Affiliated Hospital of Hubei University of Arts and Science, 136 Jingzhou Street, Xiangyang, Hubei 441021, P.R. China

E-mail: gy8c63@163.com

Key words: low-density lipoprotein, small vessel disease, logistic regression analysis existence of CSVD. To reduce the occurrence of adverse events in clinical practice, we can perform early intervention in SVD by decreasing the level of LDL, improving the endothelial function of small vessels and applying the anti-inflammation and nerve-protection methods.

\section{Introduction}

In China, we are facing a rapidly increasing aging population, and this contributes to a gradual increase in the incidence of cerebral vascular diseases. Currently, the etiology and relevant mechanism of small vessel disease (SVD) remains unclear. In clinical practice, the treatment of small vessel is in need of improvement, especially those that could not be correctly diagnosed during imagology. Consequently, it often leads to a delay in treatment and increases the risk for patients. This condition may lead to transient ischemic attack (TIA), cerebral hemorrhage, infarction, vascular cognitive impairment, affective and gait disorder, or even decreased general functions (1). Therefore, early intervention and prophylaxis of SVD are considered to be significant parameters for the prognosis of the disease (2).

Generally, TIA refers to transient insufficiency of blood supply occuring in the carotid artery or vertebro-basilar arterial system, causing local cerebral ischemia, and a sudden, transient and reversible neurological dysfunction (3). This can only last for several minutes from onset and can disappear after approximately $30 \mathrm{~min}$. However, if the duration is over $2 \mathrm{~h}$, the patient may suffer from mild neurologic impairment, and CT and MRI imaging results show the signs of cerebral ischemia. TIA is frequently observed in individuals aged 34-65 years, and those above 65 years account for $25.3 \%$, with male patients surpassing the female ones (4). The onset of TIA is usually short, and mostly caused by altering the supine position, excessive exercise, or sudden rotation or extension of neck $(3,4)$.

In this study, we investigated the correlation between the low-density lipoprotein (LDL) and TIA caused by cerebral small vascular disease (CSVD) and analyzed the effect of LDL on the TIA caused by CSVD.

\section{Materials and methods}

Inclusion and exclusion criteria. Inclusion criteria for patients enrolled in the CSVD patient group were: i) patients 
Table I. History of baseline diseases and clinical data of enrolled patients (mean \pm SD).

\begin{tabular}{lcccccc}
\hline Group & $\begin{array}{c}\text { Sex } \\
(\text { male/female })\end{array}$ & Age & $\begin{array}{c}\text { Cases } \\
(\mathrm{n})\end{array}$ & $\begin{array}{c}\text { Weight } \\
(\mathrm{kg})\end{array}$ & $\begin{array}{c}\text { Height } \\
(\mathrm{cm})\end{array}$ & BMI \\
\hline Patient group & $76 / 63$ & $54.3 \pm 16.4$ & 139 & $70.2 \pm 21.5$ & $169.7 \pm 20.4$ & $30.85 \pm 14.64$ \\
Control group & $61 / 46$ & $46.5 \pm 17.3$ & 107 & $68.4 \pm 19.8$ & $160.2 \pm 15.3$ & $28.63 \pm 16.71$ \\
F-value & & 0.82 & - & 0.79 & 0.84 & 0.63 \\
P-value & & $>0.05$ & - & $>0.05$ & $>0.05$ & $>0.05$ \\
\hline
\end{tabular}

aged 55-90 years; ii) patients with MRI or CT results showing white matter lesion (WML) and/or lacunar infarction (LI); iii) patients with no acute cortical infarction; iv) patients whose imagological results showed no non-lacunar infarction $(>15 \mathrm{~mm})$ and $\mathrm{v})$ patients who signed the written informed consent.

Exclusion criteria. Exclusion criteria for the study were: i) patients with cardiac infarction (confirmed with echocardiography); ii) patients with intracranial or extracranial macrovascular diseases; iii) patients with pulmonary infection or infection of urinary system; iv) patients with a history of severe craniocerebral trauma or intracranial tumor; v) patients with heart, liver, kidney or lung failure; vi) patients with any type of diseases that could lead to death; vii) patients with WML caused by non-ischemic factors, such as poison, genetic variability, infection, demyelinating disease, metabolic diseases, or hydrocephalus and viii) non-cooperative patients and those with a history of mental illnesses.

Inclusion criteria of patients that were enrolled in the control group were: i) patients who had risk factors associated with cerebral vascular diseases as well as stroke-like symptoms, such as dizziness, headache, onset of epilepsy, inarticulate speech or weakness of limbs but with normal results of MRI examination of head and ii) patients who signed the written informed consent. Exclusion criteria were: patients with infection or malignant diseases.

We enrolled 139 patients into the patient group. According to the MRI and/or CT of head, the patients were further divided into two subgroups: the WML group $(n=86)$ and the LI group $(n=53)$. We also enrolled 107 subjects into the control group. All participants and their families voluntarily accepted the conditions of this study. This study was approved by the Ethics Committee of the Affiliated Hospital of Hubei University of Arts and Science. Signed written informed consents were obtained from all participants before the study.

Clinical data and data collection. Inpatients and outpatients who were admitted to the hospital between September 2012 and September 2015 were enrolled. These cases were divided into the patient and control groups to investigate the correlation between the level of LDL and CSVD-induced TIA. Basic clinical data are presented in Table I. An experienced physician in the department of neurology was assigned to collect the detailed history of the disease and physical examinations including the patient's age, sex, height, weight, BMI, smoking and drinking history, history of hypertension, diabetes mellitus and heart disease.
Diagnostic criteria. After admission, patients were subjected to head MRI examination, including T1- and T2-weighted image, T2 fluid attenuated inversion recovery (FLAIR) and diffusion weighted imaging (DWI). Fasting blood glucose and lipid levels, liver and kidney function, and prothrombin chart were determined. Blood routine, CRP and regular electrocardiographic examination were conducted. For patients with aortic stenosis, we performed cerebral angiography examination, and for patients with cardiac thrombus, echocardiography was carried out.

\section{Imagological examination}

Imagological examination of SVD. After admission, patients received the MRI and/or CT examinations. The results were independently analyzed by two physicians in the imaging department to identify the types of lesion, i.e., the WML and LI and corresponding imaging diagnoses were given. Imaging diagnosis and relevant analysis was conducted independently from the general clinical data.

WML. Uniform or slightly decreased T1WI signal, highly enhanced T2WI signal or a low signal density in CT, unclear rim, no significant mass effect, patch-shaped, or mutually fused, uneven signals, border between the corpus callosum and septum pellucidum not affected, and diameters $>5 \mathrm{~mm}$. Most of the lesions were distributed in the frontal, temporal and parietal-occipital lobe, basal ganglia and infratentorial region (Fig. 1A-F).

LI. Imagological manifestation: clear rim, diameters of 3-15 $\mathrm{mm}$, and regions with the characteristics of cerebrospinal fluid (CSF) on MRI. In CT and T1WI, low intensity/signal was identified, and was similar to the features of CSF in previous LI. Thus, enhanced signal presented in diffusion-weighted images was used for diagnosis in case of the newly developed LI. LI was mainly distributed in the midline, such as deep cerebral hemispheres and brainstem (bilateral thalamus, basal ganglia, periventricular white matter and subcortical white matter) (Fig. 1G-I).

Mini-mental status examination (MMSE). The scale was 1 point for each correct answer, and 0 for wrong answers, 9 points for inappropriate answer, and 8 points for patients who refused to answer or did not understand the question. In adding up the total scores, 8 and 9 points were all recorded as 0 . The maximum score was 30 points. During the examination, the education levels and dementia were also considered. Therefore, elderly patients were judged as dementia if they met the following conditions: illiterate, score $<17$ points; primary school, score $<20$ points and high school or above, score 
A

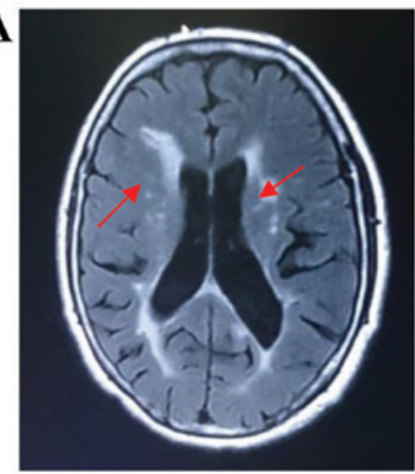

D

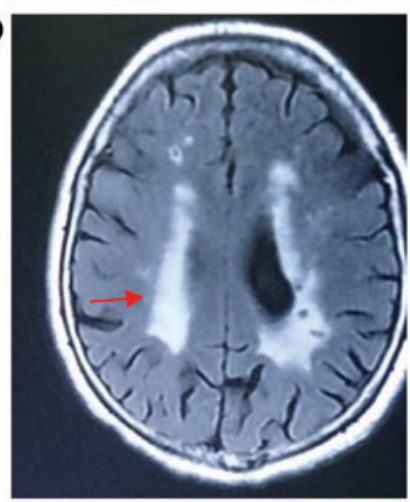

G

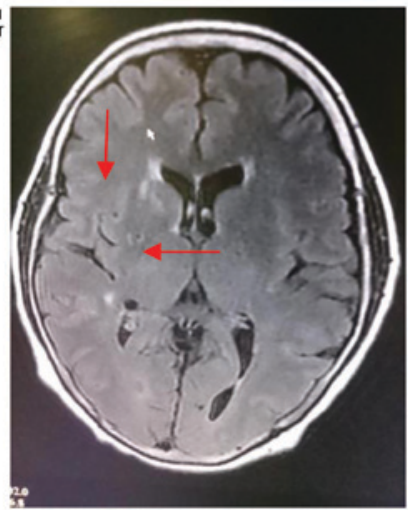

B

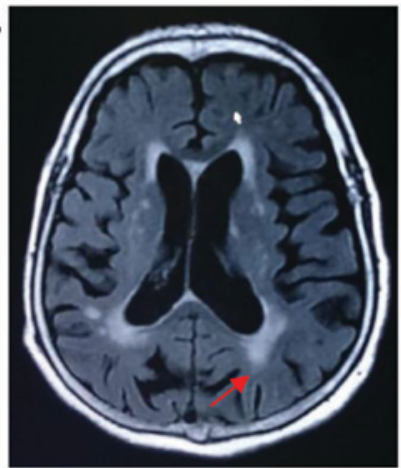

E

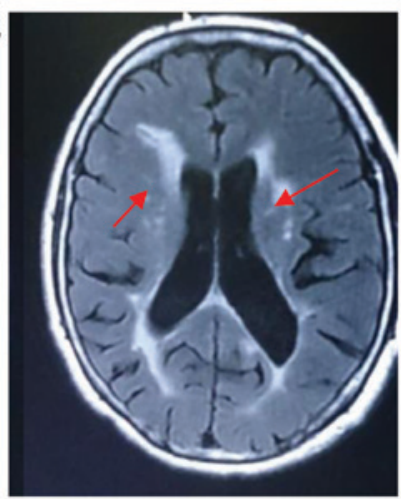

H

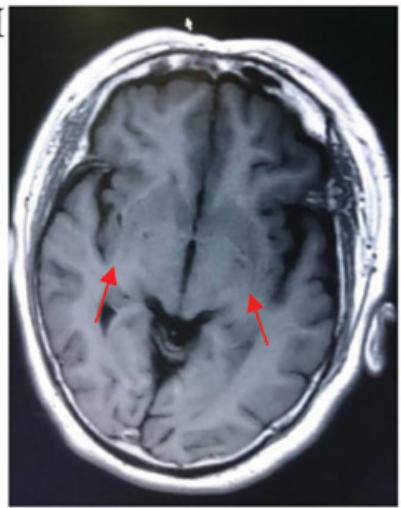

C

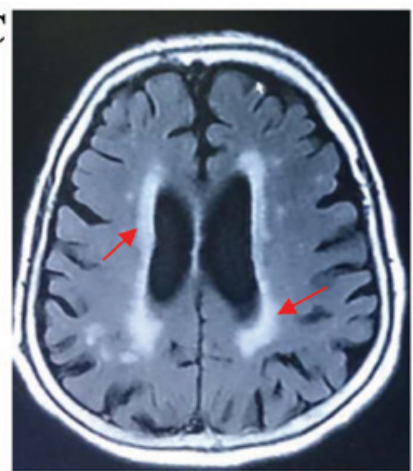

F

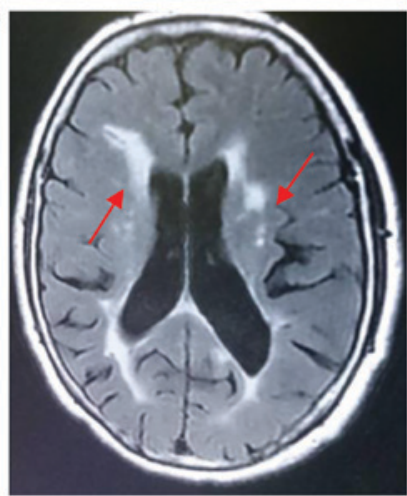

I

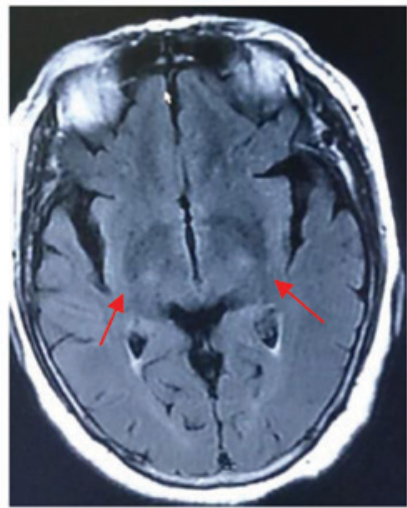

Figure 1. MRI images for CSVD. (A) (MRI FLAIR): right cerebral hemisphere (i.e., left part of the figure): leukoaraiosis (arrow), and acute cerebral infarction in left cerebral hemisphere (i.e., right part of the figure). Corresponding patient with acute cerebral infarction. (B) (MRI FLAIR): leukoaraiosis (arrow). Corresponding patient with TIA. (C) (MRI FLAIR): leukoaraiosis (arrow). Corresponding patient with TIA. (D) (MRI FLAIR): leukoaraiosis (arrow). Corresponding patient with TIA. (E) (MRI FLAIR): right cerebral hemisphere (i.e., left part of the figure): leukoaraiosis (arrow), and acute cerebral infarction in left cerebral hemisphere (i.e., right part of the figure). Corresponding patient with acute cerebral infarction. (F) (MRI FLAIR): right cerebral hemisphere (i.e., left part of the figure): leukoaraiosis (arrow), and acute cerebral infarction in left cerebral hemisphere (i.e., right part of the figure). (E and F) for the same corresponding patient with acute cerebral infarction. (G) (MRI FLAIR): LI (arrow). Corresponding patient with TIA. (H) (MRI FLAIR): bilateral LI (arrow). Corresponding patient with TIA. (I) (MRI FLAIR): bilateral LI (arrow). Corresponding patient with TIA. CSVD, cerebral small vascular disease; FLAIR, fluid attenuated inversion recovery; TIA, transient ischemic attack; LI, lacunar infarction.

$<24$ points. The mental status of patient was judged by the following criteria: 27-30 points, normal; 21-26 points, mild; 10-20 points, moderate and 0-9 points, severe.

Montreal cognitive assessment (MoCA). Detection items included: visuospatial, executive and naming ability, attention, language, abstraction, delayed recall, and orientation, with a total of 30 points; 1 point was added for an education duration $<12$ years for calibration. Higher scores represented stronger cognitive ability, and patients with scores $<26$ points were considered as normal.

Statistical analysis. Statistical analysis was performed using SPSS 20.0 (SPSS, Chicago, IL, USA). Measurement data were presented as (means \pm standard deviation), and ANOVA or t-test was used for intergroup comparison. Count data were presented as percentage, and Chi-square test was used for intergroup comparison. For each risk factor, multi-factor logistic regression analysis and correlation analysis of paired material were carried out. $\mathrm{P}<0.05$ indicated that the difference was statistically significant.

\section{Results}

Statistics of categorized test for risk factors. We summarized clinically collected results of physical and laboratory examinations, including blood pressure and glucose, total cholesterol (TC), low-density lipoprotein cholesterol (LDL-C), 
Table II. Statistics of categorized test for risk factors of patients in each group (mean \pm SD).

\begin{tabular}{|c|c|c|c|}
\hline Indexes & Group & Cases & Detection results \\
\hline \multirow[t]{5}{*}{ Fasting blood glucose $(\mathrm{mmol} / \mathrm{l})$} & Control group & 107 & $5.68 \pm 7.51$ \\
\hline & WML group & 86 & $6.32 \pm 3.3$ \\
\hline & LI group & 53 & $5.87 \pm 4.6$ \\
\hline & F-value & - & 0.62 \\
\hline & P-value & - & $>0.05$ \\
\hline \multirow[t]{5}{*}{ Systolic pressure (mmHg) } & Control group & 107 & $119.3 \pm 25.3$ \\
\hline & WML group & 86 & $136.7 \pm 13.5$ \\
\hline & LI group & 53 & $128.2 \pm 22.8$ \\
\hline & F-value & - & 0.94 \\
\hline & P-value & - & $>0.05$ \\
\hline \multirow[t]{5}{*}{ Diastolic pressure (mmHg) } & Control group & 107 & $92.12 \pm 9.6$ \\
\hline & WML group & 86 & $87.81 \pm 10.2$ \\
\hline & LI group & 53 & $95.63 \pm 15.7$ \\
\hline & F-value & - & 0.72 \\
\hline & P-value & - & $>0.05$ \\
\hline \multirow[t]{5}{*}{ LDL-C (mmol/l) } & Control group & 107 & $1.58 \pm 2.67$ \\
\hline & WML group & 86 & $3.15 \pm 1.21$ \\
\hline & LI group & 53 & $4.65 \pm 0.86$ \\
\hline & F-value & - & 1.95 \\
\hline & P-value & - & $<0.05$ \\
\hline \multirow[t]{5}{*}{ HDL-C (mmol/l) } & Control group & 107 & $1.45 \pm 1.22$ \\
\hline & WML group & 86 & $0.97 \pm 0.63$ \\
\hline & LI group & 53 & $1.25 \pm 0.57$ \\
\hline & F-value & - & 0.83 \\
\hline & P-value & - & $>0.05$ \\
\hline \multirow[t]{5}{*}{$\mathrm{Cr}(\mu \mathrm{mol} / \mathrm{l})$} & Control group & 107 & $88.52 \pm 16.82$ \\
\hline & WML group & 86 & $79.42 \pm 27.21$ \\
\hline & LI group & 53 & $90.16 \pm 25.33$ \\
\hline & F-value & - & 0.37 \\
\hline & P-value & - & $>0.05$ \\
\hline \multirow[t]{5}{*}{ TG (mmol/l) } & Control group & 107 & $1.32 \pm 0.81$ \\
\hline & WML group & 86 & $2.52 \pm 1.53$ \\
\hline & LI group & 53 & $2.87 \pm 1.36$ \\
\hline & F-value & - & 2.76 \\
\hline & P-value & - & $<0.05$ \\
\hline \multirow[t]{5}{*}{ Smoking duration (year) } & Control group & 107 & $7.62 \pm 3.8$ \\
\hline & WML group & 86 & $6.51 \pm 1.5$ \\
\hline & LI group & 53 & $8.42 \pm 1.6$ \\
\hline & F-value & - & 0.82 \\
\hline & P-value & - & $>0.05$ \\
\hline \multirow[t]{5}{*}{ Smoking amount (/day) } & Control group & 107 & $15.27 \pm 10.36$ \\
\hline & WML group & 86 & $18.48 \pm 9.68$ \\
\hline & LI group & 53 & $10.25 \pm 7.83$ \\
\hline & F-value & - & 0.98 \\
\hline & P-value & - & $>0.05$ \\
\hline \multirow[t]{5}{*}{ Drinking duration (year) } & Control group & 107 & $5.87 \pm 4.81$ \\
\hline & WML group & 86 & $5.77 \pm 6.74$ \\
\hline & LI group & 53 & $7.46 \pm 2.21$ \\
\hline & F-value & - & 0.34 \\
\hline & P-value & - & $>0.05$ \\
\hline \multirow[t]{4}{*}{ Drinking amount (ml/day) } & Control group & 107 & $108.26 \pm 22.64$ \\
\hline & WML group & 86 & $125.47 \pm 12.35$ \\
\hline & LI group & 53 & $116.35 \pm 23.56$ \\
\hline & F-value & - & 0.94 \\
\hline
\end{tabular}


Table II. Continued.

\begin{tabular}{|c|c|c|c|}
\hline Indexes & Group & Cases & Detection results \\
\hline & $\mathrm{P}$-value & - & $>0.05$ \\
\hline \multirow[t]{5}{*}{ Age (years) } & Control group & 107 & $38.7 \pm 11.2$ \\
\hline & WML group & 86 & $53.6 \pm 5.3$ \\
\hline & LI group & 53 & $58.2 \pm 7.7$ \\
\hline & F-value & - & 5.84 \\
\hline & P-value & - & $<0.05$ \\
\hline \multirow[t]{5}{*}{$\mathrm{TC}(\mathrm{mmol} / \mathrm{l})$} & Control group & 107 & $4.8 \pm 3.65$ \\
\hline & WML group & 86 & $8.7 \pm 2.24$ \\
\hline & LI group & 53 & $6.8 \pm 1.24$ \\
\hline & F-value & - & 1.68 \\
\hline & P-value & - & $<0.05$ \\
\hline
\end{tabular}

WML, white matter lesion; LI, lacunar infarction; LDL-C, low-density lipoprotein cholesterol; Cr, creatinine; TG, triglyceride; TC, total cholesterol.

Table III. MMSE and MoCA examination.

\begin{tabular}{lccc}
\hline Group & Cases & MMSE score & MoCA score \\
\hline Control group & 107 & $28.38 \pm 0.82$ & $27.32 \pm 1.24$ \\
WML group & 86 & $21.27 \pm 1.78$ & $22.47 \pm 1.35$ \\
LI group & 53 & $21.25 \pm 2.18$ & $20.35 \pm 1.56$ \\
F-value & - & 19.38 & 20.03 \\
P-value & - & 0.017 & 0.012 \\
\hline
\end{tabular}

MMSE, mini mental status examination; MoCA, Montreal cognitive assessment; WML, white matter lesion; LI, lacunar infarction.

HDL-C, creatinine (Cr), total triglyceride (TG), smoking and drinking capacity and duration, and performed the statistical analysis. Results indicated that LDL-C, TG and TC levels in the LI group were significantly higher than those in the other two groups, but ages in the control group were significantly lower than those in the other two groups with statistically significant differences $(\mathrm{p}<0.05)$ (Table II).

MMSE and MoCA examination. We conducted MMSE and MoCA examinations on all patients and the results revealed that scores in WML and LI groups were significantly lower than those in the control group and differences were statistically significant $(\mathrm{p}<0.05)$. No significant difference was detected when the LI group was compared with the WML group ( $\mathrm{p}>0.05)$ (Table III).

Logistic regression analysis between CSVD and risk factors. We collected and summarized all possible risk factors for CSVD-induced TIA, including fasting glucose, lipid level, $\mathrm{Cr}$ level, smoking and drinking amount and duration. The logistic regression analysis and correlation analysis of paired material revealed that the serum level of LDL-C was correlated to the CSVD-induced TIA $(\mathrm{OR}=1,321)$ and the difference was statistically significant despite the enrollment of TG into the multivariable analysis $(\mathrm{p}<0.05)$ (Table IV).

\section{Discussion}

Small cerebral vessels refer to the small perforator arteries, small arteries, capillary and small veins with diameters of 40-200 $\mu \mathrm{m}$ that are distributed inside the brain. Small cerebral vessels constitute the basic units that supply blood for cerebral tissues, and play a significant role in maintaining the cerebral function. The small cerebral vessels, consisting of endothelial cells and a few smooth muscle cells, are characterized by poor vascular elasticity and are usually susceptible to injuries due to lack of outer layer in histological structure (1-3). Small thrombus could be easily developed due to the increase in blood viscosity caused by augmentation of red blood cells or blood fat, causing endothelial injuries, and secondarily leading to the TIA and/or cerebral infarction. The main pathological manifestations include fibrinoid necrosis and amyloidosis, which may lead to cerebral hemorrhage and infarction (4-7). Generally, CSVD refers to the syndrome consisting of the clinical, imagological and pathological manifestations caused by various lesions. CSVD can be divided into two categories: i) acute neurological impairment, which is manifested by stroke, deep cerebral infarction and cerebral hemorrhage with acute onset, rapid progression, poor prognosis and a high mortality and disability rate and ii) chronic and progressive neuropsychic dysfunction, including vascular cognitive impairment, affective disorder, decreased comprehension ability, memory impairment, gait disorder and general function reduction (8-12). Imagological features are typically manifested by LI, leukoaraiosis, microbleeding, which can be easily missed or misdiagnosed (13-15). In the International Stroke Conference and European Stroke Conference held in 2006, experts introduced a document titled 'Little strokes, big trouble' (1), which was repeated in the International Stroke Conference and European Stroke Conference held in 2008, suggesting that CSVD has gradually gained the attention of international medical communities. 
Table IV. Analysis of correlation of CSVD-induced TIA with LDL-C and TG.

\begin{tabular}{|c|c|c|c|c|c|c|}
\hline \multirow[b]{2}{*}{ CSVD } & \multicolumn{2}{|c|}{ LDL-C } & \multicolumn{2}{|c|}{ TG } & \multirow[b]{2}{*}{$\mathrm{r}$} & \multirow[b]{2}{*}{ P-value } \\
\hline & Positive $^{\mathrm{a}}$ & Negative & Positive $^{\mathrm{b}}$ & Negative & & \\
\hline Positive & 139 & 0 & 125 & 11 & 0.108 & $<0.05$ \\
\hline Negative & 23 & 86 & 17 & 90 & 0.024 & $<0.05$ \\
\hline
\end{tabular}

CSVD positive refers that patients diagnosed as LI and/or WML. ${ }^{a}$ LDL-C positive indicates that LDL-C was $>4.14 \mathrm{mmol} / \mathrm{l}$. ${ }^{\mathrm{b}} \mathrm{TG}$ positive refers to the level of TG in plasma $>1.65 \mathrm{mmol} / \mathrm{l}$. CSVD, cerebral small vascular disease; TIA, transient ischemic attack; LDL-C, low-density lipoprotein cholesterol; TG, triglyceride; WML, white matter lesion; LI, lacunar infarction.

Currently, the consensus is that CSVD-induced TIA is caused by various factors, and lipid metabolism disorders is one of the risk factors of CSVD. However, the harm posed by the increase of LDL level is the most serious compared to all other factors. Therefore, CSVD can be prevented by controlling the level of LDL in clinical practice (7). Prior studies often investigated CSVD and the abnormal level of blood fat independently, and partially neglected the effect of other risk factors on this disease. Therefore, it is imperative to clarify the exact relationship between the CSVD and other related risk factors, in order to perform early intervention in this disease.

Studies conducted on small-sample groups showed that CSVD is mainly correlated with the age, hypertension, hyperlipidemia and atherosclerosis, but the specific mechanism remained unknown (2). Results obtained from prior studies suggested that the treatment with statins could effectively inhibit the development of SVD (3-6). These results suggested that there may be a certain association between the abnormal lipid metabolism and SVD. In the present study we evaluated a larger group, and discovered a positive correlation between LDL-C level and the incidence rate of CSVD. Currently, lipid infiltration theory has been confirmed in experimental and clinical practice, suggesting that the increase in LDL can promote cholesterol to pass through the arterial wall and localized aggregation can induce the macrophages and smooth muscle cells to transform into the foam cells by consuming the lipid (16). This is also considered an important mechanism of atherosclerotic plaque formation. LDL-C plays an important role in the formation of atherosclerosis by the following mechanism: after LDL is transported into the lower layer of arterial endothelium, it is transformed into ox-LDL through oxidative modification, which is transported into the macrophages by the scavenger receptor on the cell membrane. Macrophages that devoured and digested the lipid are transformed into the foam cells, constituting the fatty streak through aggregation. Cholesterol can be released after the rupture of fatty streak, forming the lipid core of atherosclerotic plaque. Previous findings revealed that the major component of LDL is LDL-3, i.e., small but intensive LDL shows the strongest effect in inducing atherosclerosis. This is mainly manifested as follows: the clearance rate of LDL is very slow through the pathway mediated by its receptor and LDL-3 adheres to the vascular wall and is transported into the endothelial cells of a vessel $(16,17)$. LDL can easily pass through the arterial wall to be devoured by the macrophages. LDL is oxidized and ox-LDL is formed. Shen et al confirmed that there was a correlation between LDL-C in low-density and cardiovascular events in patients with chronic renal diseases (16). Currently, ox-LDL is considered a relatively sensitive index for predicting the small vascular lesions. Thus, we consider that LDL-C may be an important risk factor in the occurrence of CSVD-induced TIA, among which the LDL in a low density shows the strongest effect.

We studied a relatively large sample, and combined the investigations of the various factors. We found that LDL is the cause for CSVD and explained the correlation between the risk factors and CSVD.

We concluded that LDL is a major risk factor affecting the onset of CSVD. We believe that CSVD should be studied in patients with hyperlipidemia. Patients should be examined by head MRI or CT in order to eliminate the probability of CSVD. In order to reduce the occurrence of adverse events in clinical practice, early intervention in SVD through decreasing the level of LDL, improving the endothelial function of small vessels and applying the anti-inflammation and nerve-protection methods should be considered.

\section{References}

1. Hachinski V: World Stroke Day 2008: 'Little strokes, big trouble'. Stroke 39: 2407-2420, 2008.

2. Pantoni L: Cerebral small vessel disease: From pathogenesis and clinical characteristics to therapeutic challenges. Lancet Neurol 9: 689-701, 2010.

3. Amarenco P, Benavente O, Goldstein LB, Callahan A, Sillesen H, Hennerici MG, Gilbert S, Rudolph AE, Simunovic L, Zivin JA, et al; Stroke Prevention by Aggressive Reduction in Cholesterol Levels Investigators: Results of the stroke prevention by aggressive reduction in cholesterol levels (SPARCL) trial by stroke subtypes. Stroke 40: 1405-1409, 2009.

4. ten Dam VH, van den Heuvel DM, van Buchem MA, Westendorp RG, Bollen EL, Ford I, de Craen AJ and Blauw GJ; PROSPER Study Group: Effect of pravastatin on cerebral infarcts and white matter lesions. Neurology 64: 1807-1809, 2005.

5. Mok VC, Lam WW, Fan YH, Wong A, Ng PW, Tsoi TH, Yeung V and Wong KS: Effects of statins on the progression of cerebral white matter lesion: Post hoc analysis of the ROCAS (Regression of Cerebral Artery Stenosis) study. J Neurol 256: 750-757, 2009.

6. Fu JH, Mok V, Lam W, Wong A, Chu W, Xiong Y, Ng PW, Tsoi TH, Yeung V and Wong KS: Effects of statins on progression of subclinical brain infarct. Cerebrovasc Dis 30: 51-56, 2010.

7. Fu JH, Lu CZ, Hong Z, Dong Q, Luo Y and Wong KS: Extent of white matter lesions is related to acute subcortical infarcts and predicts further stroke risk in patients with first ever ischaemic stroke. J Neurol Neurosurg Psychiatry 76: 793-796, 2005.

8. Fu JH, Wong K, Mok V, Hu X, Xiong Y, Chen Y, Tang WK, Chen X, Wong A, Chu W, et al: Neuroimaging predictors for depressive symptoms in cerebral small vessel disease. Int $\mathbf{J}$ Geriatr Psychiatry 25: 1039-1043, 2010. 
9. Jokinen H, Gouw AA, Madureira S, Ylikoski R, van Straaten EC, van der Flier WM, Barkhof F, Scheltens P, Fazekas F, Schmidt R, et al; LADIS Study Group: Incident lacunes influence cognitive decline: The LADIS study. Neurology 76: 1872-1878, 2011.

10. van Dijk EJ, Prins ND, Vrooman HA, Hofman A, Koudstaal PJ and Breteler MM: Progression of cerebral small vessel disease in relation to risk factors and cognitive consequences: Rotterdam Scan study. Stroke 39: 2712-2719, 2008.

11. Greenberg SM: Small vessels, big problems. N Engl J Med 354: 1451-1453, 2006.

12. Pantoni L, Basile AM, Pracucci G, Asplund K, Bogousslavsky J, Chabriat H, Erkinjuntti T, Fazekas F, Ferro JM, Hennerici M, et al: Impact of age-related cerebral white matter changes on the transition to disability - The LADIS study: Rationale, design and methodology. Neuroepidemiology 24: 51-62, 2005.

13. Pantoni L: Cerebral small vessel disease: from pathogenesis and clinical characteristics to therapeutic challenges. Lancet Neurol 9: 689-701, 2010.
14. Patel B and Markus HS: Magnetic resonance imaging in cerebral small vessel disease and its use as a surrogate disease marker. Int J Stroke 6: 47-59, 2011.

15. Fazekas F, Chawluk JB, Alavi A, Hurtig HI and Zimmerman RA: MR signal abnormalities at $1.5 \mathrm{~T}$ in Alzheimer's dementia and normal aging. AJR Am J Roentgenol 149: 351-356, 1987.

16. Shen H, Xu Y, Lu J, Ma C, Zhou Y, Li Q, Chen X, Zhu A and Shen G: Small dense low-density lipoprotein cholesterol was associated with future cardiovascular events in chronic kidney disease patients. BMC Nephrol 17: 143, 2016.

17. Annema W and von Eckardstein A: Dysfunctional high-density lipoproteins in coronary heart disease: Implications for diagnostics and therapy. Transl Res 173: 30-57, 2016. 